\title{
Correlation Analysis of Dominant Eye and Refractive Error Between Monozygotic Twins
}

\author{
Jian Jiang ${ }^{\prime}$ \\ Jingjing $\mathrm{Xu}^{\prime}$ \\ Yuwen Wang' \\ Jingwei Zheng ${ }^{2}$ \\ Dan $\mathrm{Xu}^{3}$ \\ 'Clinical Optometry Center, Eye Hospital \\ of Wenzhou Medical University, Wenzhou, \\ Zhejiang Province, 325027, People's \\ Republic of China; ${ }^{2}$ Clinical Research \\ Center, Eye Hospital of Wenzhou Medical \\ University, Wenzhou, Zhejiang Province, \\ 325027, People's Republic of China; \\ ${ }^{3}$ School of Ophthalmology \& Optometry, \\ Wenzhou Medical University, Wenzhou, \\ Zhejiang Province, 325027, People's \\ Republic of China
}

\begin{abstract}
Objective: This study aims to investigate the correlation of dominant eye and refractive error between monozygotic twins.

Methods: The data of dominant eye and refractive error of 13 pairs of monozygotic twins who were treated at the Optometry Clinic were collected. The paired chi-square test and Kappa consistency test were used to analyze the data of dominant eye between monozygotic twins, while the paired $t$ test and Pearson correlation analysis were adopted to analyze the refractive error data. SPSS version 22.0 software was used to analyze the above statistics.

Results: In the Kappa consistency test of dominant eye between monozygotic twins, Kappa value $=0.451, \mathrm{p}=0.052, \mathrm{p}>0.05$, and in the paired chi-square test, $\mathrm{p}=0.250$. In the spherical equivalent paired $t$ test for the right eye of monozygotic twins, $\mathrm{t}=1.491$ and $\mathrm{p}=0.162$, while in the spherical equivalent paired $t$ test for the left eye, $\mathrm{t}=0.753$ and $\mathrm{p}=0.466$. In the spherical equivalent correlation analysis of monozygotic twins, for the spherical equivalent refraction of the right eye, the Pearson correlation results were $r=0.901$ and $p=0.00$, and for the spherical equivalent refraction of the left eye, the Pearson correlation results were $r=$ 0.971 and $\mathrm{p}=0.00$.

Conclusion: The difference in the chi-square test of dominant eye is not statistically significant between monozygotic twins, but dominant eye and refractive error are correlated to some extent. It is suggested that heredity may be the main determinant of the dominant eye. There is no difference in refractive error between identical twins which are highly correlated, however.
\end{abstract}

Keywords: refractive error, dominant eye, monozygotic twins/identical twins, heredity, genes

\section{Introduction}

The optical properties of the eye are determined by several factors, including corneal curvature (CC), anterior chamber depth (ACD), refractive power of lens, and axial length (AL). Refractive error can be broadly defined as an optical state. ${ }^{1}$ The incidence of refractive error is rising rapidly, especially in urban areas of Southeast Asia. ${ }^{2,3}$ Therefore, refractive error is listed by the Global Initiative as one of the top five eye diseases to be eliminated by $2020 .{ }^{4}$ Many studies have shown that myopia is a complex ophthalmopathy with both genetic and environmental factors. ${ }^{5-7}$ In epidemiological studies, close working is consistently identified as a major environmental factor for myopia. ${ }^{8}$ In addition, the genetic factors of refractive error have been confirmed. The risk of myopia in children with nearsighted parents is greater than that in children with non-nearsighted parents. In recent years, a large number of twin-based studies have shown that genetic factors
Correspondence: Dan Xu

School of Ophthalmology \& Optometry,

Wenzhou Medical University, Wenzhou,

Zhejiang Province, 325027, People's

Republic of China

Tel +8657786689937

Email xudammmc@163.com 
are associated with refractive error. ${ }^{9-13}$ Dominant eye is also known as ocular dominance, leading eye, and master eye. It is divided into right dominant eye, denoted as right type (R type), and left dominant eye, denoted as left type (L type). Most dominant eyes are genetically determined or formed in childhood, which is relatively difficult to reverse.

Many studies have shown that different nationalities have different ratios of dominant eye. The right dominant eye has a higher probability of distribution in the population than the left dominant eye, which has a significant difference. Most of the previous dominant eye studies focused on the correlation of myopia, astigmatism, depth perception and pupil size to study the relationship of the dominant eye in anisometropia, as well as the changes of the dominant eye before and after the correction of refractive error and refractive surgery. ${ }^{14-16}$ In China, Wu found that the consistency of refractive state is very high in monozygotic twins. ${ }^{17}$ The twin method carries out a comparison based on the internal correlation between identical twins and non-identical twins with genetic similarity. They have the same genes. Compared with nonidentical twins, identical twins have a higher internal correlation. A total of 13 pairs of identical twins who were treated in the Outpatient Department of the Optometry Center of our hospital in recent years were enrolled in this study. Through the measurement of refractive error degree and the detection of dominant eye between twins, the correlation of dominant eye and refractive error between identical twins was analyzed.

\section{Materials and Methods General Data}

The data on the degree of dominant eye and refractive error of 13 pairs of identical twins who were treated at the Outpatient Department of the Optometry Center of the Eye Hospital of WMU in recent years were collected. The subjects included 4 pairs $(\mathrm{n}=8)$ of male twins and 9 pairs $(n=18)$ of female twins. The overall age range was 10-32 years, with a mean age of $14.08 \pm 5.69$ years. Inclusion criteria: (1) corrected visual acuity $\geq 1.0$; and (2) clear refracting media. Exclusion criteria: (1) systemic and severe congenital diseases, such as hypertension, diabetes, and heart disease; (2) organic eye lesions, including cataracts, strabismus, and fundus lesions; and 3) a history of major ocular trauma and a history of internal and external eye surgery.

\section{Examination Methods}

All the twins were required to undergo an ocular physical examination, including corrected visual acuity, slit-lamp anterior segment examination, and direct fundus examination, and subjects who did not meet the inclusion criteria were excluded. The dominant eyes of 13 pairs of twins (n = 26) were measured by the card-hole method and recorded. Based on computer optometry, the subjective refraction was carried out by using a comprehensive refractometer. The prescription for glasses was obtained by trying on. The prescription for glasses was used as the degree of refractive error for the analysis between twins.

\section{Data Grouping}

The degree of refractive error of the right eye vs the right eye and the left eye vs the left eye was discussed in groups between twins. The dominant eye was analyzed between twins.

\section{Statistical Analyses}

SPSS version 22.0 software was used for the analyses. The paired chi-square test and the Kappa consistency test were used to analyze the data of dominant eye between different twins. The refractive error data were analyzed by using the paired $t$ test and Pearson correlation. All the statistical results were considered to be statistically significant $(\mathrm{p}<0.05)$.

\section{Results}

\section{Paired $t$ test for Spherical Refractive Error in Twins}

Results of the paired $t$ test for spherical refractive error in the right eye were $t=1.414, p=0.183$, and those for spherical refractive error in the left eye were $\mathrm{t}=0.606, \mathrm{p}=$ 0.556 . The difference in the degree of spherical refractive error was not statistically significant between twins.

\section{Paired $t$ test for Astigmatism in Twins}

Results of the paired $t$ test for astigmatism of the right eye were $t=0.874, p=0.399$, and those for astigmatism of the left eye were $t=0.473, p=0.645$. The difference in astigmatism of both eyes was not statistically significant between twins.

\section{Paired $t$ test for Spherical Equivalent in Twins}

Results of the paired $t$ test for spherical equivalent of the right eye were $t=1.491, p=0.162$, and those for spherical 
equivalent of the left eye were $\mathrm{t}=0.753, \mathrm{p}=0.466$

Regardless of whether it was the right eye or left eye, the difference in the degree of equivalent refractive error was not significant between twins.

\section{Correlation Analysis Results of Spherical Refractive Error in Twins}

The Pearson correlation results for the degree of spherical refractive error of the right eye were $r=0.967, p=0.00$. The difference in correlation of spherical refractive error of the right eye was statistically significant between twins. The Pearson correlation results for the degree of spherical refractive error of the left eye were $r=0.973, p=0.00$. The difference in correlation of spherical refractive error of the left eye was statistically significant between twins (Figure 1).

\section{Correlation Analysis Results of Astigmatism in Twins}

The Pearson correlation results for the degree of astigmatism of the right eye were $\mathrm{r}=0.859, \mathrm{p}=0.00$. The difference in spherical correlation of the right eye was statistically significant between twins. The Pearson correlation results for the degree of astigmatism of the left eye were $r=0.785, p=0.01$. The difference in astigmatism of the left eye was statistically significant between twins.

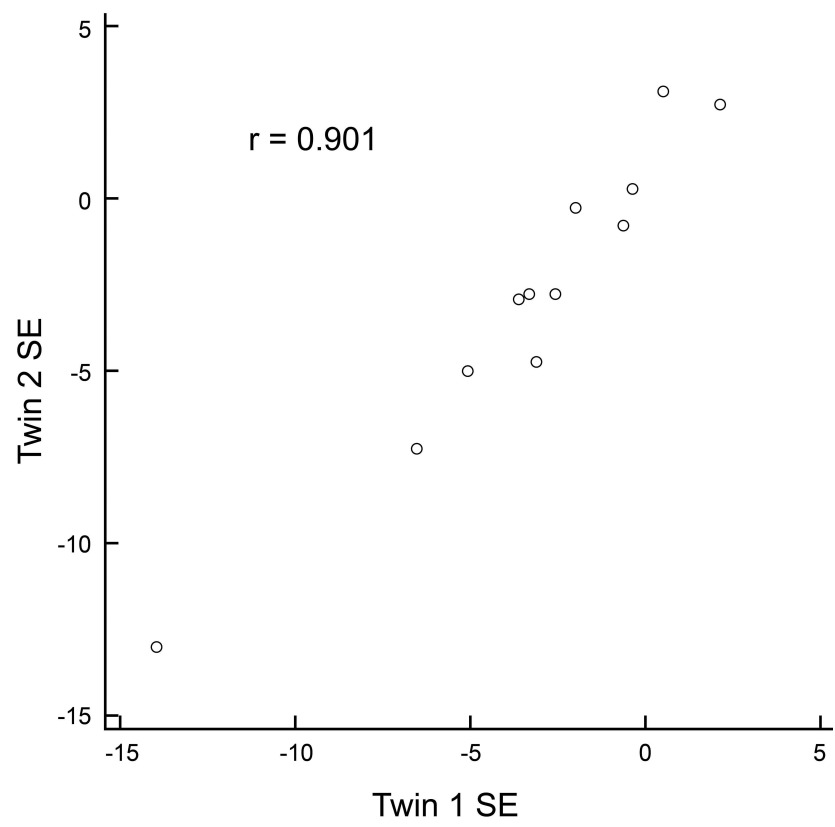

Figure I Scatter diagram of equivalent spherical mirror.

\section{Correlation Analysis Results of Spherical} Equivalent in Twins

The Pearson correlation results for the degree of spherical equivalent of the right eye were $r=0.901, p=0.00$. The difference in correlation of degree of spherical equivalent of the right eye was statistically significant between twins. The Pearson correlation results for the degree of spherical equivalent of the left eye were $r=0.971, p=0.00$. The difference in correlation of degree of spherical equivalent of the right eye was statistically significant between twins.

\section{Correlation Analysis of Dominant Eye in Twins}

Results of the Kappa consistency test and chi-square test were $0.451, p=0.052$. Although the numerical deviation was small, it also indicated $p>0.05$. It showed that the consistency statistics of dominant eye were not statistically significant between twins.

\section{Discussion}

In human vision, one eye is usually stronger than the other. Not only the vision, but also more visual information of the brain is from the eye. This eye is the dominant eye. Many studies have shown that different ethnic groups have different ratios, and a right dominant eye has a higher probability of distribution in the population than a left dominant eye. The difference in the distribution is significant. In about $60 \%$ of cases, the dominant eye is the right eye, while in about $30 \%$ of cases, the dominant eye is the left eye. The dominant eye of about $5 \%$ of patients can alter between the left and right eyes in the clinical examination. Among patients with refractive error, there is no significant change in the dominant eye before and after correction ${ }^{18}$ and after refractive surgery. ${ }^{19}$ Among patients with anisometropia, there is no obvious correlation between the dominant eye and the high degree eye, and the low degree eye is not necessarily the dominant eye. ${ }^{16}$ In a study of the relationship between corrected visual acuity and dominant eye, the eye with better corrected visual acuity is not necessarily the dominant eye. Moreover, the right or left dominant eye may affect the refractive state in the future. It has been found that the size of congenital astigmatism may affect the determination of dominant eye after birth. In the study of dominant eye and depth perception, it has been found that the inequality in binocular function exists in depth perception. The dominant eye plays an important role in depth 
perception, which is the influence of the dominant eye on depth perception formed in the acquired social practice activities, showing a trait of age since it increases with age.

In a study of the relationship between pupil size and dominant eye, it was found that the dominant eye is associated with the pupil size. It is considered that the dominant eye is often determined by congenital factors. Most studies show that the dominant eye is often formed at birth and is relatively stable. However, in animal experiments and clinical studies of patients with monocular amblyopia, it was found that the dominant eye can be transferred to the other eye due to certain acquired causes, such as dominant eye trauma or dominant eye cover. This shows that the dominant eye still has a certain degree of acquired plasticity.

Monozygotic twins have the same genes and genetic consistency. Using monozygotic twins for clinical controls or clinical trials can exclude genetic factors, and the results are more reliable. Some previous clinical observations have found that there is strong consistency in the degree of refractive error between identical twins, namely, the similarity of refractive error state is very high between twins. Wu's study in China found that the correlation of refractive error between twins is very high; Clinical data showed no significant difference in refractive error between twins. ${ }^{17}$ The refractive errors of the two groups were consistent. There was a significant difference in refractive error between different twins. Furthermore, the occurrence and progression of myopia are mostly related to environmental factors.

Our study aims to determine whether the dominant eye relies on innate and genetic factors. Among 13 pairs of identical twins with dominant eye, we found that 10 pairs of identical twins have a completely consistent dominant eye (right or left), of which 8 pairs have a right dominant eye and two pairs have a left dominant eye. Therefore, right dominant eye accounts for $80 \%$ and left dominant eye for $20 \%$. This is in line with the results of a previous study that in the majority of cases, the right eye is the dominant eye.

Monozygotic twins have the same genes, and the refractive state is consistent between them. With this characteristic, different treatment measures are adopted clinically between twins. The effectiveness of treatment factors has been studied. For example, in the observation and study of the popular methods of myopia progression control, we can use identical twins.
The sample size of this paper is really small because there are few such clinical patients. However, the results of this paper show that the dominant eye is highly correlated with heredity, which is not consistent with the previous literature.

In addition, we found that there are great differences in diopter between different twins, indicating that the acquired factors of myopia are greater, and we need to strengthen the prevention and control of myopia after birth.

\section{Ethics Approval and Consent to Participate}

This study was conducted with approval from the Ethics Committee of Wenzhou Medical University. Written informed consent was obtained from all participants. This study was conducted in accordance with the declaration of Helsinki. The parent have provided informed consent for participants under the age of 18 years.

\section{Funding}

This work was supported by the Wenzhou Science and Technology Bureau Project (Y20180171).

\section{Disclosure}

The authors report no conflicts of interest in this work.

\section{References}

1. Curtin BJ. The myopias. basic science and clinical management. Harper Row Philadelphia. 1985.

2. Woo WW, Lim KA, Yang H, et al. Refractive errors in medical students in Singapore. Singapore Med J. 2004;45(10):470-474.

3. Morgan IG, Rose KA, Smith W, Mitchel L. Evidence for an "epidemic" of myopia. Ann Acad Med Singapore. 2004;33:541-543.

4. Pararajasegaram R. VISION. 2020-The Right to Sight: from strategies to action. Am J Ophthalmol. 1999;128:357-358.

5. Young TL, Ronan SM, Alvear AB. A second locus for familial high myopia maps to chromosome 12q. Am $J$ Hum Genet. 1998;5:1419-1424. doi:10.1086/302111

6. Young TL, Ronan SM, Drahozal LA, et al. Evidence that a locus for familial high myopia maps to chromosome 18p. Am J Hum Genet. 1998;63:109-119. doi:10.1086/301907

7. Naiglin I, Gazagne C, Dallongeville F, et al. A genome wide scan for familial high myopia suggests anovel locus on chromosome $7 \mathrm{q} 36$. J Med Genet. 2002;39:118-124. doi:10.1136/jmg.39.2.118

8. Saw SM, Tan SB, Fung D, et al. IQ and the association with myopia in children. Invest Ophthalmol Vis Sci. 2004;45:2943-2948. doi:10.1167/iovs.03-1296

9. Sorsby A, Sheridan M, Leary GA. Refractions and its components during growth of the eye after the age three. Med Res Council Special Rep Ser. 1962;301:1-18.

10. Valluri S, Minkovitz JB, Budak K, et al. Comparative corneal topography and refractive variables in monozygotic and dizygotic twins. Am J Ophthalmol. 1999;127:158-163. doi:10.1016/S0002-9394(98) 00319-5 
11. Lyhne N, Sjolie AK, Kyvik KO, Green A. The importance of genes and environment for ocular refraction and its determiners: a population based study among 20-45 year old twins. $\mathrm{Br}$ J Ophthalmol. 2001;85:1470-1476. doi:10.1136/bjo.85.12.1470

12. Chen CJ, Cohen BH, Diamond EL. Genetic and environmental effects on the development of myopia in Chinese twin children. Ophthalmic Paediatr Genet. 1985;6:353-359.

13. Hammond CJ, Snieder H, Gilbert CE, Spector TD. Genes and environment in refractive error: the twin eye study. Invest Ophthalmol Vis Sci. 2001;42:1232-1236.

14. Huang J, Su B, Wu H, et al. Relationship between perceptual dominant eye and myopic refractive parallax. Chin J Pract Ophthalmol. 2017;35(06):601-605.

15. Hoshikawa R, Ito M, Yano T, Tsutsui K, Sato T, Shimizu K. Association between ocular dominance and anisometropic hyperopia. Am Orthopt J. 2016;66(1). doi:10.3368/aoj.66.1.107
16. Duan W, Yang Y, Wang L. Distribution and relationship of dominant eye in myopia and astigmatism population. Int $J$ Ophthalmol. 2015;15(11):1974-1976.

17. Wu Y. Correlation of refractive status of twins. Chin Pract Med. 2010;5(32):34.

18. Wang W, Jin H, Tian J, et al. Comparison of refractive error correction before and after the thumb method, Worth four-point lamp method and cavity method for measuring the dominant eye. Pract Clin Med. 2012;13(11):77-78+108.

19. Tan Y, Xu L, Wang L, Lu X, Zhou X. Analysis of the dominant eye before and after LASIK correction for myopia. Int $J$ Ophthalmol. 2009;9(08):1548-1550.

\section{Publish your work in this journal}

The International Journal of General Medicine is an international, peer-reviewed open-access journal that focuses on general and internal medicine, pathogenesis, epidemiology, diagnosis, monitoring and treatment protocols. The journal is characterized by the rapid reporting of reviews, original research and clinical studies across all disease areas. The manuscript management system is completely online and includes a very quick and fair peer-review system, which is all easy to use. Visit http://www.dovepress.com/ testimonials.php to read real quotes from published authors. 